\title{
Recensión bibliográfica
}

Subramanian, I., Farahnik, J., y Mischley, L. (2020). Synergy of pandemics-social isolation is associated with worsened Parkinson severity and quality of life. Nature, 6 (28), 1-8, https://doi.org/10.1038/s41531-020-00128-9.

Colavitta, M. F.*

La Dra. Indu Subramanian es médica neuróloga, especializada en trastornos del movimiento. Recibida en la Universidad de California, Los Ángeles (UCLA), actualmente ejerce como directora en uno de los Southwest Parkinson's Disease Research, Education and Clinical Centers (PADRECCs), los cuales son considerados centros de excelencia en el tratamiento de la Enfermedad de Parkinson (EP). Situados en seis estados de EEUU, en ellos se desempeñan reconocidos especialistas en trastornos del movimiento, neurocirujanos, psiquiatras, psicólogos, investigadores, educadores y otros expertos en EP. Con un enfoque en la calidad de vida de los adultos mayores con este padecimiento, así como la de sus cuidadores, el interés radica en el tratamiento integral. La Dra. Subramnian también se ha especializado en técnicas de medicina integrativa, como acupuntura, yoga, Tai Chi y mindfulness, con el objetivo de ofrecer un acercamiento más holístico hacia los pacientes.
En el marco de la pandemia por COVID-19, Subramanian y su equipo han llevado a cabo un estudio con 1527 pacientes con EP idiopática, revelando el impacto de la soledad sobre la severidad de esta enfermedad, potenciada por los efectos del aislamiento sanitario. Ellos destacan la importancia de un abordaje más comprensivo de la realidad de los pacientes con EP. Sentirse solo contribuye a la complejidad del cuadro, y cobra un sentido fundamental tras los notorios efectos deletéreos del aislamiento preventivo por la pandemia, que se suman al sentimiento de soledad y la tendencia al aislamiento que suelen presentar las personas con EP. Por ello, los autores resaltan en este artículo, la necesidad de aprovechar los abordajes nuevos y alternativos para ayudar a mejorar la calidad de vida de los pacientes, así como de sus familias y cuidadores, quienes suelen verse también afectados por el efecto de la pandemia y el sentimiento de soledad en esta población.

\footnotetext{
*Estudiante avanzada de Psicología. Pontificia Universidad Católica Argentina. Centro de Investigaciones en Psicología y Psicopedagogía. Adscripta en la línea de investigación sobre deterioro cognitivo en adultos mayores. Mail de contacto: mariaflorenciacol@ hotmail.com

DOI: https://doi.org/10.46553/RPSI.17.34.2021.p87-91
}

Fecha de Recepción: 20 de octubre de 2021 Fecha de Aceptación: 20 de octubre de 2021 
La tesis principal de este estudio es que el aislamiento social, entendiendo por esto a la falta de integración de los individuos en su entorno - como vivir solo, poseer pocas redes sociales, y tener un mínimo contacto social-, constituye un factor de riesgo para un empeoramiento de la salud e incremento de la mortalidad en la EP, y que este cobra una relevancia fundamental en la situación de pandemia por COVID-19. Se entrevistaron individuos con EP idiopática con el objetivo de evaluar si el aislamiento social se vio relacionado con la severidad de la sintomatología del cuadro y la calidad de vida de pacientes y cuidadores en el contexto de pandemia. Se recabaron datos acerca del sentimiento de soledad, en relación a una variedad de factores tales como la amistad, el estado civil y la performance social.

En la población con EP, hay una multitud de razones para el aislamiento social, independientemente de la situación sanitaria global actual. Estas razones se ven principalmente asociadas a estigmas asociados a esta enfermedad. Por vergüenza ante sus síntomas motores como el temblor o las discinesias, la incontención urinaria, o ante la dificultad para caminar sin la ayuda de un bastón, estas personas tienden a recluirse socialmente. De la misma manera, las expresiones faciales, la disartria y la disfonía, contribuyen a la dificultad en la comunicación verbal y no verbal, lo cual refuerza la evitación de los espacios sociales. También la apatía y la depresión contribuyen a un descenso en la motivación para frecuentar espacios compartidos, según el artículo.

Durante la era de COVID-19, el distanciamiento puede empeorar el aislamiento social, y perjudicar a esta población, vulnerabilizándolos aún más. Subramanian y colaboradores destacan que la soledad puede ocurrir en tres dimensiones independientes: la soledad emocional (deseo de un compañero confidente y cercano), la soledad relacional (deseo de compañía social y amistad) y colectiva (deseo de una comunidad o red de apoyo que comparta los propios intereses). El sentimiento de soledad en cualquiera de estas tres, incrementa la susceptibilidad de los efectos del estrés y constituye un predictor de la pobreza de la respuesta inmune. Asimismo, afecta el rendimiento cognitivo y puede generar ansiedad, fatiga e irritabilidad. Estos resultados se constatan en otros estudios, que a su vez han reportado que el distrés psicológico se vio incrementado en la población con EP durante la pandemia por COVID-19, lo cual llevó a un empeoramiento de los síntomas motores y no motores, viéndose perturbada, en consecuencia, la calidad de vida (Suzuki et al., 2021).

Los resultados del estudio de Subramanian et al. arrojan que la soledad se vio significativamente correlacionada con una menor calidad de vida en las personas con EP, y una peor y más rápida evolución del cuadro. El sentimiento de soledad fue la variable que más se relacionó con la progresión de la enfermedad. Se puede concluir que el aislamiento social genera efectos nocivos en la calidad de vida, que se ven potenciados con las medidas sanitarias en respuesta a la pandemia, y que se necesita más consideración en relación a esto al momento de abordar el tratamiento de cada paciente. En otra investigación también realizada en el marco de la pandemia, Saluja et al. (2021) han señalado la necesidad del seguimiento del impacto del aislamiento 
sobre esta población, considerando el aumento de los reportes, tanto de los pacientes como de sus cuidadores, en cuanto al empeoramiento motor y no-motor durante la pandemia.

Debe tenerse en cuenta que los pacientes pueden sentirse solos a pesar de estar rodeados de gente, así como también pueden existir muchos con tendencias más introvertidas, que se sentirán más cómodos sin tanta gente alrededor, y, por lo tanto, no reportarán sentirse solos. Este estudio tuvo en cuenta el sentimiento de soledad, y no la soledad en sí, para la obtención de datos. Esta precaución en el análisis de los resultados permite dar cuenta de la importancia de la subjetividad de las personas para poder mensurar su calidad de vida y orientar el tratamiento a un objetivo ajustado a su propia perspectiva de la situación individual. Es decir, que a pesar de que todos los pacientes estén diagnosticados con la misma enfermedad, la cuantificación de los datos no puede menoscabar la concepción idiosincrática del padecimiento de cada persona ante ese diagnóstico y su curso.

Subramanian et al., en este trabajo, remiten a un nuevo concepto en vistas al tratamiento en este estado de soledad: la prescripción social. Sugieren el uso de recursos y actividades en la comunidad para ayudar al paciente a desarrollar conexiones sociales saludables. Por ejemplo, mediante clases grupales de determinados ejercicios, el tratamiento grupal de condiciones específicas, sesiones de arte-terapia grupales, o actividades comunitarias como la jardinería y la cocina. La idea de realizar programas de apoyo psicosocial y comunitario para el paciente, sus familiares y cuidadores ya habría sido remarcado por otros autores algunos años previos a la pandemia. Sigcho Vivanco (2017) remarca la validez de charlas psicoeducacionales y programas de vinculación comunitaria para favorecer el intercambio entre pares mediante la generación de experiencias compartidas, encontrando y generando una nueva red de apoyo social, para contribuir al manejo de la enfermedad y a una mejor calidad de vida para los pacientes y su entorno familiar.

Asimismo, el estudio de Subramanian et al. también propone nuevas intervenciones en pos de lograr este mejor manejo del tratamiento y una mejora en la calidad de vida. Entre estas se encuentran herramientas provenientes de las nuevas tecnologías que permiten a los usuarios verse unos a otros mediante una pantalla, y que pueden resultar muy útiles para el contacto social, aún en el entorno de pandemia. En este trabajo, la telemedicina surge como un concepto interesante. Esta constituye una herramienta que permite seguir brindando atención médica, así como mantener el contacto con los médicos. Su aplicación mejora la calidad de vida y aumenta la eficacia y adherencia a los tratamientos, a la vez que sana el perjuicio del aislamiento social. Una de las principales formas de telemedicina, la cual la Dra. Subramanian también aplica en su labor diaria y en los PADRECCs, es la rehabilitación virtual. Esta estimula, a través de un formato lúdico, el movimiento en pacientes con EP, y les permite practicar la motricidad, motivación y aprendizaje en ambientes seguros. Estas sugerencias coinciden con lo reportado en un estudio italiano, cuyos resultados proponen la combinación de tecnologías disponibles, al demostrar ser herramientas útiles, fáciles de aplicar, y que pueden tener un impacto 
considerable en la salud de los pacientes con habilidades limitadas, especialmente en un contexto crítico como lo es la pandemia actual (Motolese, 2020).

La pandemia ha contribuido a poder reconsiderar la importancia de uno de los aspectos no motores que más pueden limitar la calidad de vida de las personas con EP y quienes las rodean: la soledad. A partir de los resultados obtenidos en este trabajo, se enfatiza la importancia de mantener a los pacientes con EP socialmente conectados, y prevenir el sentimiento subjetivo de soledad, especialmente durante estos tiempos de distanciamiento social preventivo, que ha generado un impacto aún más negativo en esta población. De todos modos, si bien la pandemia podrá verse concluida en un tiempo futuro, esto no implica un olvido de este aspecto al momento de tratar cada caso. El sentimiento subjetivo de soledad constituye uno de los factores de riesgo más importantes sobre el bienestar, por lo cual, considerando que ya existe de por sí un aislamiento debido a conductas evitativas frecuentes en la EP, la pandemia por COVID-19 y el trabajo de la Dra. Subramanian y su equipo han sabido recalcar la importancia de tener en cuenta este fenómeno al tratar con todas las personas que padecen esta enfermedad, así como de sus cuidadores. Este artículo grafica particularmente el notable poder de la conexión social en esta enfermedad neurodegenerativa.

En conclusión, se requiere la toma de conciencia sobre la relevancia del sentimiento de soledad y la vulnerabilidad en la que posiciona a la persona con EP. Ante este panorama, se hace evidente la necesidad de implementación de políticas públicas que se orienten a la prevención de estos aspectos, tan importantes como la sintomatología que constituye el cuadro, porque aporta de la misma forma o más a su calidad de vida. El primer paso es descubrir las variables determinantes y el segundo, generar espacios, dispositivos y herramientas que permitan la modificación efectiva de las mismas, ayudando a que las personas construyan una mejor vida a pesar de la EP.

Desde la psicología, podemos colaborar a la creación de intervenciones psicoeducativas, charlas, y espacios que se orienten a la apertura de estas personas a la sociedad, para que se vean incluidos, apoyados y acompañados, y que disminuya el sentimiento de soledad y de vergüenza o temor a la exposición social. Este artículo abre un espacio esencial a la disciplina psicológica dado que la EP no se trata simplemente de un trastorno del movimiento, sino una enfermedad multifactorial que implica la imbricación de variados factores biológicos, genéticos y ambientales, de entre los cuales el posicionamiento subjetivo ante el padecimiento y la calidad de vida serán los más factibles de moldear para ayudar a atravesar la situación de la mejor manera posible. Sería deseable ayudar a promover un estilo de vida más adaptativo para evitar una progresión más desfavorable. De esta manera, mantener a las personas con EP socialmente conectadas y prevenir el sentimiento subjetivo de soledad en tiempos de distanciamiento social, con técnicas virtuales de apoyo y alternativas terapéuticas que involucren un enfoque social, deberían ser explorados. 


\section{Referencias}

Motolese, F., Magliozzi, A., Puttini, F., Rossi, M., Capone, F., Karlinski, K., StarkInbar, A., Yekutieli, Z., Di Lazzaro, V., Marano, M. (2020) Parkinson's Disease remote patient monitoring during the COVID-19 lockdown. Frontiers in Neurology, 11, 1-7. DOI: https://doi.org/10.3389/ fneur.2020.567413.

Saluja, A., Parihar, J., Garg, D., Dhamija, R. K. (2021). The impact of COVID-19 pandemic on disease severity and quality of life in Parkinson's Disease. Journal of Indian Academy Neurology, 24(2), 217-226.
Sigcho Vivanco, M. G. (2017). Enfoque desde la perspectiva social, familiar e intercultural en pacientes con Enfermedad de Parkinson [Tesis de grado]. Universidad Técnica de Machala.

Suzuki, K., Numao, A., Komagamine, T., Haruyama, Y. Kawasaki, A., Funakoshi, K., Fujita, H., Suzuki, S., Okamura, M. (2021). Impact of the COVID-19 Pandemic on the Quality of Life of patients with Parkinson's Disease and their caregivers: A single-center survey in Tochigi Prefecture. Journal of Parkinson's Disease, 11(3), 10471056 . 